\title{
Morphologic Response and Tumor Shrinkage as Early Predictive Markers in Unresectable Colorectal Liver Metastases
}

\author{
TOSHIKI MASUISHI ${ }^{1}$, HIROYA TANIGUCHI ${ }^{1}$, TETSUYA ETO ${ }^{2}$, AZUSA KOMORI ${ }^{1}$, \\ SEIICHIRO MITANI ${ }^{1}$, HIROKO HASEGAWA ${ }^{1}$, YUKIYA NARITA ${ }^{1}$, MAKOTO ISHIHARA ${ }^{2}$, \\ TSUTOMU TANAKA ${ }^{2}$, SHIGENORI KADOWAKI ${ }^{1}$, TAKASHI URA ${ }^{1}$, MASASHI ANDO ${ }^{1}$, \\ MASAHIRO TAJIKA ${ }^{3}$, MOTOO NOMURA ${ }^{4}$, YOZO SATO ${ }^{5}$, HIDEYUKI MISHIMA $^{6}$ and KEI MURO ${ }^{1}$ \\ ${ }^{1}$ Department of Clinical Oncology, Aichi Cancer Center Hospital, Nagoya, Japan; \\ ${ }^{2}$ Department of Gastroenterology, Tsuchiura Kyodo General Hospital, Tsuchiura, Japan; \\ ${ }^{3}$ Department of Endoscopy, Aichi Cancer Center Hospital, Nagoya, Japan; \\ ${ }^{4}$ Department of Therapeutic Oncology, Kyoto University Graduate School of Medicine, Kyoto, Japan; \\ ${ }^{5}$ Department of Diagnostic and Interventional Radiology, Aichi Cancer Center Hospital, Nagoya, Japan; \\ ${ }^{6}$ Cancer Center, Aichi Medical University, Nagakute, Japan
}

\begin{abstract}
Background/Aim: Anti-EGFR antibodies or bevacizumab comprise first-line treatment for patients with RAS wild-type colorectal liver metastases (CLM). Which marker better predicts efficacy, early tumor shrinkage or morphologic response, still remains unclear. Patients and Methods: We retrospectively evaluated 155 patients with KRAS exon 2 wild-type CLM treated with bevacizumab (BEV group) or anti-EGFR antibodies (EGFR group). Three radiologists independently assessed early tumor shrinkage (ETS) and early optimal morphologic response (EOMR) from baseline and first follow-up CT scan. Results: Patients with ETS had longer progression-free survival (PFS) than those without ETS [hazard ratio $(H R)=0.69$ ] and ETS tended to be observed in the EGFR group, while patients with EOMR had longer PFS than those without EOMR $(H R=0.64)$ and EOMR tended to be observed in the BEV group. Conclusion: Among patients with KRAS exon 2 wild-type CLM, EOMR and ETS may predict better PFS, especially in patients treated with bevacizumab and anti-EGFR antibodies, respectively.
\end{abstract}

Patients with $R A S$ wild-type and $R A S$-mutant metastatic colorectal cancer (CRC) have different treatment strategies. Anti-epidermal growth factor receptor (EGFR) antibodies are

Correspondence to: Hiroya Taniguchi, Department of Clinical Oncology, Aichi Cancer Center Hospital, 1-1 Kanokoden, Chikusaku, Nagoya, Aichi 464-8681, Japan. Tel: +81 527626111, Fax: +81 527642967, e-mail: h.taniguchi@aichi-cc.jp

Key Words: Colorectal cancer, early predictive marker, morphologic response, tumor shrinkage. administered to patients with $R A S$ wild-type mCRC because studies have shown that $R A S$ mutations predict a lack of response to anti-EGFR antibodies (1). Bevacizumab or antiEGFR antibodies in combination with fluoropyrimidine, plus either oxaliplatin or irinotecan, is the first-line standard treatment for patients with $R A S$ wild-type mCRC. However, not all patients respond to this regimen.

Recently, surrogate markers of the survival benefit of these agents have been reported. Early tumor shrinkage (ETS), which may be defined as either $\geq 20 \%$ (2) or $\geq 30 \%$ (3) decrease in tumor size from the baseline, has been shown to be a useful surrogate marker of survival benefit in patients with $K R A S$ wild-type mCRC who have been treated with anti-EGFR antibodies. Another surrogate marker, optimal morphologic response, has been reported to be a predictor of benefit in patients with colorectal liver metastases (CLM) who have been treated with bevacizumab (4). Morphologic response is a measure of the transformation of liver metastases into homogeneous and hypo-attenuating lesions with well-defined borders after treatment (5). However, an optimal morphologic response was defined as the best response during first-line treatment in these previous reports $(4,5)$. In addition, little has been reported on the usefulness of morphologic response in CLM with extrahepatic metastases. Therefore, no reports are available on whether morphologic response is useful as an early surrogate marker of efficacy in CLM with extrahepatic metastases. In addition, there are no reports concerning the association between tumor shrinkage and morphologic response as an early surrogate marker of efficacy.

In the present study, we analyzed morphologic response or tumor shrinkage at an early time point after the initiation of first-line chemotherapy in $K R A S$ exon 2 wild-type mCRC 
patients with liver metastases, regardless of whether extrahepatic metastases were present or not in these patients.

\section{Materials and Methods}

Patients. We performed a retrospective review of a prospectively maintained database of mCRC patients who underwent palliative chemotherapy at the Aichi Cancer Center Hospital and the Tsuchiura Kyodo General Hospital from April 2007 to December 2014. Eligibility criteria included the following: 1) histologically confirmed, unresectable colorectal adenocarcinoma; 2) KRAS exon 2 wild-type tumors; 3) first-line treatment comprising fluoropyrimidines, and either oxaliplatin or irinotecan, and either bevacizumab or anti-EGFR antibodies (cetuximab or panitumumab); 4) liver metastases, regardless of the presence of extrahepatic metastases; 5) Eastern Cooperative Oncology Group performance status (ECOG PS) of 0-2; and 6) adequate bone marrow, hepatic, and renal function.

All the patients provided written informed consent for their treatment. The protocol for the present study was reviewed and approved by the Institutional Review Boards of the Aichi Cancer Center Hospital (approval number: 2015-1-059) and the Tsuchiura Kyodo General Hospital (approval number: 467).

Evaluation of imaging. All the patients underwent enhanced CT at the start of chemotherapy and then approximately every 2-3 months during follow-up to evaluate tumor response. Three radiologists blinded to the clinical data independently reviewed the CT images from the scans taken at baseline and at the first follow-up visit. Morphologic response of the liver metastases was assessed according to morphologic criteria $(4,5)$, and tumor shrinkage of the liver metastases was assessed according to the Response Evaluation Criteria in Solid Tumors (RECIST) version 1.1 (6). Discrepancies between radiologists were resolved by consensus review.

Morphologic criteria were defined as follows: group 3, heterogeneous attenuation and a thick, poorly defined tumor-liver interface; group 1, homogeneous low attenuation with a thin, sharply defined tumor-liver interface; group 2, intermediate morphology that could not be rated as either group 3 or group 1 (4, 5). Early optimal morphologic response (EOMR) was defined as a change in morphology from group 3 or 2 at baseline CT to group 1 at the first follow-up CT. In patients with multiple liver metastases, morphologic response was assigned on the basis of the response observed in the majority of tumors. ETS was defined as a $\geq 30 \%$ decrease from baseline to the first follow-up CT scan, in the longest diameter tumor measurement.

Statistical analysis. Progression-free survival (PFS) was defined as the time from the first administration of treatment to the first radiological or clinical observation of disease progression or death due to any cause, whichever occurred first. Overall survival (OS) was defined as the time from the first treatment administration until death due to any cause or censored at the last follow-up date. The median PFS and OS were estimated using the Kaplan-Meier method. Patient characteristics were compared using the Fisher's exact test. PFS and OS were compared using the log-rank test, and hazard ratio (HR) and 95\% confidence interval (95\% CI) were estimated using the Cox proportional hazards model. All statistical analyses were performed using JMP version 10 (SAS Institute, Cary, NC, USA). All statistical tests were two-sided, and $p$-values $<0.05$ were considered to be statistically significant.

\section{Results}

Patient characteristics. A total of 285 patients with KRAS exon 2 wild-type mCRC were treated with fluoropyrimidines and oxaliplatin or irinotecan in combination with bevacizumab or anti-EGFR antibodies as first-line chemotherapy at the two institutions included in this study. Patients excluded from the retrospective study included 100 patients without liver metastases, 29 patients without baseline or follow-up enhanced CT scans, and one patient with an ECOG PS 3. Patient characteristics of the 155 patients included in the retrospective study are listed in Table I. More patients were treated with oxaliplatin in combination with bevacizumab than with oxaliplatin in combination with anti-EGFR antibodies (95\% vs. 42\%). The bevacizumab treated group had fewer patients with lymph nodes metastases (25\% vs. $42 \%)$ and fewer patients with high white blood cell (WBC) counts $(8 \%$ vs. 24\%) compared to the group treated with anti-EGFR antibodies. Almost all other baseline characteristics were similar between patients treated with bevacizumab and those treated with anti-EGFR antibodies.

Frequency of EOMR and ETS according to treatment with targeted therapies. EOMR was observed in 39 (42\%) of 93 patients treated with bevacizumab and $10(16 \%)$ of 62 patients treated with anti-EGFR antibodies $(p=0.0008)$. ETS was observed in $41(44 \%)$ of 93 patients treated with bevacizumab and $36(58 \%)$ of 62 patients treated with antiEGFR antibodies $(p=0.10)$ (Table II).

Association between ETS or EOMR and survival in the whole study population. The median follow-up time of patients in the study was 29.3 months. PFS of patients with ETS was significantly longer than that of patients without ETS (non-ETS) (ETS vs. non-ETS, 13.0 vs. 10.3 months; $\mathrm{HR}=0.69 ; 95 \% \mathrm{CI}=0.50-0.96 ; p=0.03$ ) (Figure 1a). ETS was also associated with better OS (ETS vs. non-ETS, $32.8 v s$. 27.1 months; HR=0.76; $p=0.13$ ). In addition, PFS of patients with EOMR was significantly longer than that of patients without EOMR (non-EOMR) (EOMR vs. non-EOMR, 14.5 vs. 10.5 months; $\mathrm{HR}=0.64 ; 95 \% \mathrm{CI}=0.44-0.90 ; p=0.01$ ) (Figure 1b). EOMR was also associated with better OS (EOMR vs. non-EOMR, 34.5 vs. 25.9 months; HR=0.70; $p=0.06)$. Among patients without EOMR, ETS was also associated with better PFS (ETS $v s$. non-ETS, $12.8 v s .8 .7$ months; HR=0.63; $p=0.03$ ), while among patients without ETS, EOMR was also associated with better PFS (EOMR $v s$. non-EOMR, 14.5 vs. 8.7 months; HR=0.52; $p=0.01$ ).

Early predictive biomarkers of survival for patients treated with anti-EGFR antibodies. The median follow-up time of patients treated with anti-EGFR antibodies was 24.3 months. Patients with ETS had significantly longer PFS compared to 
Table I. Patient characteristics.

\begin{tabular}{|c|c|c|c|c|c|c|c|}
\hline \multirow{2}{*}{$\begin{array}{l}\text { Characteristics } \\
\text { Age }\end{array}$} & \multicolumn{2}{|c|}{$\begin{array}{c}\text { All } \\
\mathrm{N}=155(\%)\end{array}$} & \multicolumn{2}{|c|}{$\begin{array}{c}\text { Bevacizumab } \\
\mathrm{N}=93(\%)\end{array}$} & \multicolumn{2}{|c|}{$\begin{array}{l}\text { Anti-EGFR antibodies } \\
\qquad \mathrm{N}=62(\%)\end{array}$} & \multirow[t]{2}{*}{$p$-Value } \\
\hline & & & & & & & \\
\hline$<65$ years & 95 & (61) & 60 & $(65)$ & 35 & $(56)$ & 0.32 \\
\hline$\geq 65$ years & 60 & (39) & 33 & (35) & 27 & (44) & \\
\hline \multicolumn{8}{|l|}{ Gender } \\
\hline Male & 96 & $(62)$ & 56 & $(60)$ & 40 & (65) & 0.62 \\
\hline Female & 59 & (38) & 37 & (40) & 22 & (35) & \\
\hline \multicolumn{8}{|l|}{ ECOG performance status } \\
\hline $0-1$ & 144 & (93) & 88 & $(95)$ & 56 & $(90)$ & 0.35 \\
\hline 2 & 11 & (7) & 5 & (5) & 6 & (10) & \\
\hline \multicolumn{8}{|l|}{ Histological type } \\
\hline Well/Moderately & 131 & (84) & 75 & $(81)$ & 56 & $(90)$ & 0.15 \\
\hline Poorly/Mucinous & 20 & (13) & 15 & (16) & 5 & (8) & \\
\hline Unknown & 4 & (3) & 3 & (3) & 1 & (2) & \\
\hline \multicolumn{8}{|l|}{ Primary tumor location } \\
\hline Right-sided colon ${ }^{\mathrm{a}}$ & 36 & (23) & 22 & (24) & 14 & (23) & 1.00 \\
\hline Left-sided colonb/rectum & 118 & (76) & 70 & (75) & 48 & (77) & \\
\hline Unknown & 1 & (1) & 1 & (1) & 0 & 0 & \\
\hline \multicolumn{8}{|l|}{ Metastases } \\
\hline Synchronous & 129 & (83) & 74 & (80) & 55 & (89) & 0.19 \\
\hline Metachronous & 26 & (17) & 19 & (20) & 7 & (11) & \\
\hline \multicolumn{8}{|l|}{ Primary tumor resection } \\
\hline Yes & 109 & (70) & 69 & (74) & 40 & $(65)$ & 0.21 \\
\hline No & 46 & $(30)$ & 24 & (26) & 22 & (35) & \\
\hline \multicolumn{8}{|l|}{ Metastatic sites } \\
\hline Liver & 155 & $(100)$ & 93 & $(100)$ & 62 & $(100)$ & - \\
\hline Lung & 60 & (39) & 37 & $(40)$ & 23 & $(37)$ & 0.87 \\
\hline Lymph node & 49 & (32) & 23 & (25) & 26 & (42) & 0.034 \\
\hline Peritoneum & 27 & (17) & 17 & (18) & 10 & (16) & 0.83 \\
\hline \multicolumn{8}{|l|}{ Liver-limited disease } \\
\hline Yes & 57 & (37) & 37 & $(40)$ & 20 & $(32)$ & 0.40 \\
\hline No & 98 & (63) & 56 & (60) & 42 & (68) & \\
\hline \multicolumn{8}{|l|}{ Number of liver metastases } \\
\hline$\leq 4$ & 59 & (38) & 38 & (41) & 21 & (34) & 0.40 \\
\hline$\geq 5$ & 96 & (62) & 55 & (59) & 41 & (66) & \\
\hline \multicolumn{8}{|c|}{ Maximum size of liver metastases } \\
\hline$<50 \mathrm{~mm}$ & 78 & $(50)$ & 51 & $(55)$ & 27 & (44) & 0.19 \\
\hline$\geq 50 \mathrm{~mm}$ & 77 & (50) & 42 & (45) & 35 & (56) & \\
\hline \multicolumn{8}{|l|}{ Cytotoxic agents } \\
\hline Oxaliplatin & 114 & (74) & 88 & (95) & 26 & $(42)$ & $<0.0001$ \\
\hline Irinotecan & 41 & (26) & 5 & (5) & 36 & (58) & \\
\hline \multicolumn{8}{|l|}{ WBC count } \\
\hline$<10,000 / \mu 1$ & 133 & (86) & 86 & $(92)$ & 47 & (76) & 0.005 \\
\hline$\geq 10,000 / \mu \mathrm{l}$ & 22 & (14) & 7 & (8) & 15 & (24) & \\
\hline \multicolumn{8}{|l|}{ ALP levels } \\
\hline$<300 \mathrm{IU} / 1$ & 55 & (35) & 32 & (34) & 23 & (37) & 0.74 \\
\hline$\geq 300 \mathrm{IU} / 1$ & 100 & (65) & 61 & (66) & 39 & (63) & \\
\hline
\end{tabular}

ECOG: Eastern Cooperative Oncology Group; WBC: white blood cell; ALP: alkaline phosphatase. aCecum, ascending colon, and right-sided transverse colon; b ${ }^{b}$ eft-sided transverse colon, descending colon, and sigmoid colon.

those without ETS (ETS vs. non-ETS, 14.9 vs. 6.8 months; $\mathrm{HR}=0.47 ; p=0.01$ ) (Figure 2). In addition, although EOMR was associated with better PFS (EOMR $v s$. non-EOMR, 20.9 vs. 10.0 months; $\mathrm{HR}=0.39 ; p=0.01)$, the number of patients with EOMR was very small $(16 \%)$.
In patients with left-sided tumor (left-sided transverse colon, descending colon, sigmoid colon, and rectum), patients with ETS had significantly longer PFS compared to those without ETS (ETS vs. non-ETS, 17.7 vs. 6.8 months; $\mathrm{HR}=0.41 ; p=0.009$ ), while in patients with right-sided tumor 
Table II. Frequency of early optimal morphologic response (EOMR) and early tumor shrinkage (ETS) according to targeted therapy.

\begin{tabular}{|c|c|c|c|}
\hline & $\begin{array}{l}\text { Bevacizumab group } \\
\qquad(\mathrm{n}=93)\end{array}$ & $\begin{array}{l}\text { Anti-EGFR group } \\
\qquad(\mathrm{n}=62)\end{array}$ & $p$-Value \\
\hline ETS & $(44 \%)$ & $(58 \%)$ & 0.10 \\
\hline EOMR & $39 \quad(42 \%)$ & $(16 \%)$ & 0.0008 \\
\hline
\end{tabular}

(cecum, ascending colon, and right-sided transverse colon), patients with ETS had similar PFS to those without ETS (ETS vs. non-ETS, 7.6 vs. 7.2 months; HR=0.76; $p=0.66$ ).

Early predictive biomarkers of survival for patients treated with bevacizumab. The median follow-up time of patients treated with bevacizumab was 30.2 months. PFS was longer for patients with EOMR compared to those without EOMR, (EOMR vs. non-EOMR, 12.8 months vs. 10.9 months; $\mathrm{HR}=0.68 ; p=0.07$ ) (Figure 3). However, ETS was not associated with better PFS (ETS vs. non-ETS, $11.2 v s .11 .8$ months; $\mathrm{HR}=0.89 ; p=0.59$ ).

\section{Discussion}

In this retrospective cohort study, among KRAS exon 2 wildtype mCRC patients with liver metastases, we showed that EOMR may predict a better therapeutic outcome, even in the absence of ETS and tended to be observed in patients treated with bevacizumab. It was also shown that ETS may predict a better therapeutic outcome, even in the absence of EOMR and tended to be observed in patients treated with anti-EGFR antibodies. Based on these results, it appears that morphologic response is an early predictive marker of therapeutic outcomes, especially in patients treated with bevacizumab and tumor shrinkage is an early predictive marker of therapeutic outcomes, especially in patients treated with anti-EGFR antibodies. To the best of our knowledge, the present study is the first to evaluate whether morphologic response and/or tumor shrinkage can serve as an early predictive marker of therapeutic outcomes in patients treated with bevacizumab or anti-EGFR antibodies.

It has been reported that an optimal morphologic response during first-line treatment is a prognostic marker in patients with CLM $(4,5,7)$. Conversely, we investigated whether a morphologic response measured at the first follow-up CT was as an early predictive marker of improved PFS and OS in patients with CLM, regardless of whether extrahepatic metastases were present or not. Early assessment of morphologic response may enable early decisions as to whether a chemotherapy regimen should be changed, and incentivize for patients to continue receiving chemotherapy treatment. However, further studies are required to evaluate the actual advantages of early decisions regarding altering chemotherapy regimens.

In most guidelines, regimens containing bevacizumab or anti-EGFR antibodies are recommended as the first-line chemotherapy for patients with $R A S$ wild-type mCRC patients. Although previous studies of patients treated with and without bevacizumab have suggested that morphologic response is a predictor of long-term outcomes (4), no reports are available on morphologic response in patients treated with anti-EGFR antibodies. Although in the present study we showed that patients treated with either bevacizumab or anti-EGFR antibodies exhibited an EOMR, the proportion of patients with an EOMR who received anti-EGFR antibodies was very low (16\%). This percentage of patients is similar to that of patients treated with cytotoxic agents not in combination with anti-EGFR antibodies or bevacizumab who exhibited an optimal morphologic response (12\%) (4). Therefore, EOMR does not appear to be an appropriate early predictive marker of therapeutic outcomes for patients treated with anti-EGFR antibodies. Consistent with previous studies in patients treated with anti-EGFR antibodies, patients with ETS had better outcomes than patients without ETS. Thus, ETS may be more appropriate as an early predictive marker of therapeutic outcomes for patients treated with anti-EGFR antibodies than EOMR.

The results of this study suggest that EOMR may be a more appropriate early predictive marker of therapeutic outcomes than ETS for patients treated with bevacizumab. Two observations support this conclusion. First, the proportion of patients showing EOMR who received bevacizumab was relatively high. As tumor angiogenesis is directly related to contrast enhancement on CT (8), bevacizumab $(9,10)$ like other angiogenesis inhibitors, can decrease attenuation on CT. Second, the present study suggested that bevacizumab treated patients with EOMR had better outcomes than patients without EOMR, regardless of whether the patients exhibited ETS.

In the present study, among patients without ETS, PFS of patients treated with bevacizumab was longer than that of patients treated with anti-EGFR antibodies (11.8 vs. 6.8 months, respectively). This finding is consistent with the results of the FIER-3 trial, which compared bevacizumab plus FOLFIRI with cetuximab plus FOLFIRI in mCRC patients with KRAS exon 2 wild-type tumors. One reason for the consistency in the results may be that among patients treated with bevacizumab, PFS of patients with EOMR was longer than that of patients without EOMR, and the proportion of patients with EOMR was higher among patients treated with bevacizumab than that among patients treated with anti-EGFR antibodies, even in the absence of ETS. 

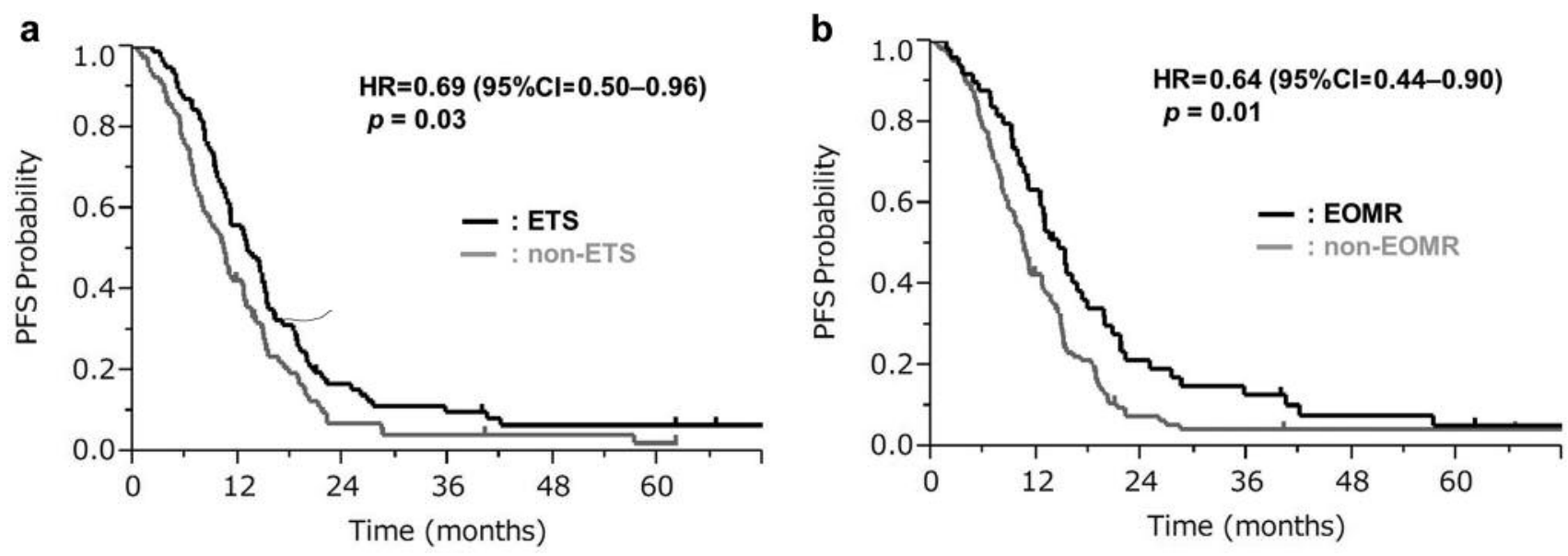

Figure 1. Kaplan-Meier curves for progression-free survival (PFS) of the whole study population. (a) PFS of patients with early tumor shrinkage (ETS) and without ETS (non-ETS). (b) PFS of patients with optimal morphologic response (EOMR) and without EOMR (non-EOMR).

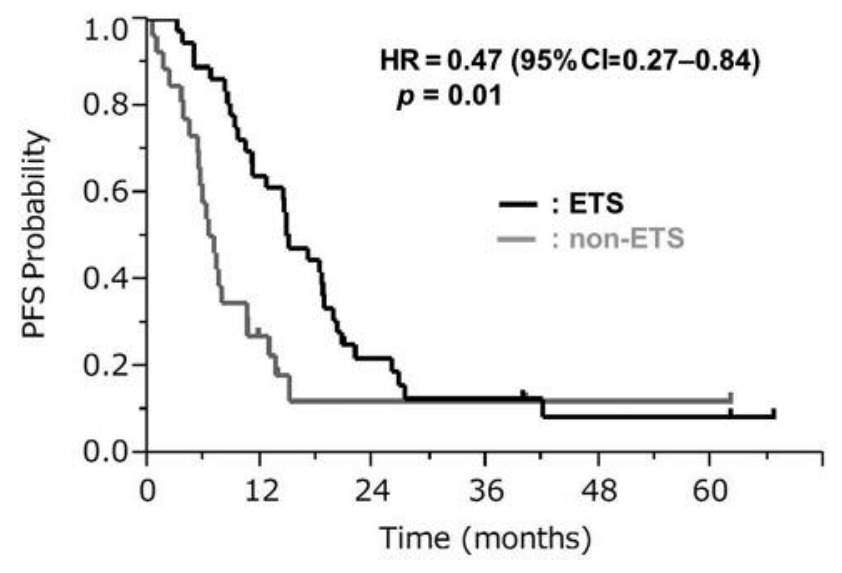

Figure 2. Kaplan-Meier curves for progression-free survival (PFS) of patients with early tumor shrinkage (ETS) and without ETS (non-ETS) among patients treated with anti-EGFR antibodies.

The present study has several limitations. First, it was a retrospective analysis. Thus, a robust conclusion cannot be drawn from our findings. However, because three radiologists, blinded to clinical data, independently reviewed the CT scans, potential bias was reduced, and the results may be a useful guide for physicians in clinical practice. Second, the sample size, i.e., the number of patients treated with antiEGFR antibodies, was small. However, the results of the present study are consistent with those of previous studies, which support the validity of our findings.

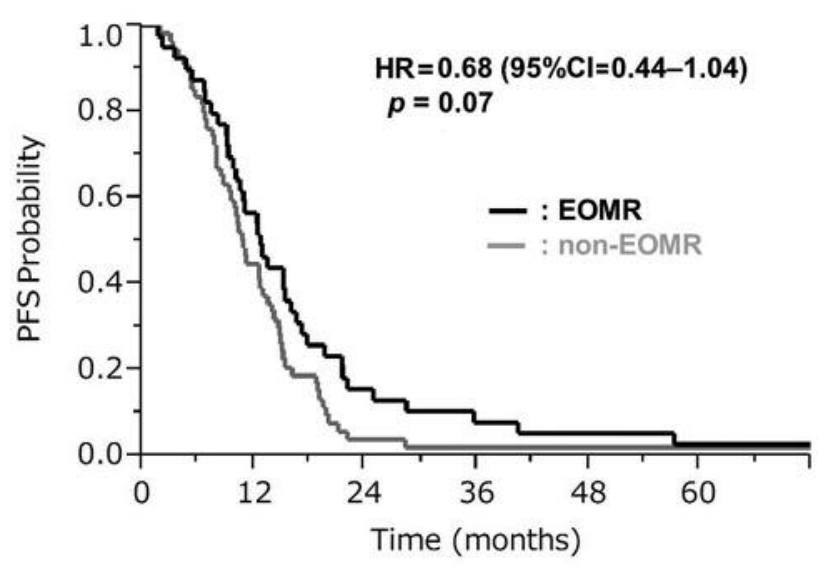

Figure 3. Kaplan-Meier curves for progression-free survival (PFS) of patients with early optimal morphologic response (EOMR) and without EOMR (non-EOMR) among patients treated with bevacizumab.

In conclusion, among KRAS exon 2 wild-type mCRC patients who have liver metastases, EOMR may predict better prognosis in patients treated with bevacizumab, even in the absence of ETS, while in patients treated with anti-EGFR antibodies, ETS may predict a better prognosis, even in absence of EOMR.

\section{Acknowledgements}

The Authors wish to thank Ms. Yumiko Kohno for reviewing the CT images in the present study. 


\section{References}

1 Douillard JY, Oliner KS, Siena S, Tabernero J, Burkes R, Barugel M, Humblet Y, Bodoky G, Cunningham D, Jassem J, Rivera F, Kocakova I, Ruff P, Blasinska-Morawiec M, Smakal M, Canon JL, Rother M, Williams R, Rong A, Wiezorek J, Sidhu R and Patterson SD: Panitumumab-FOLFOX4 treatment and RAS mutations in colorectal cancer. N Engl J Med 369: 1023-1034, 2013.

2 Piessevaux H, Buyse M, Schlichting M, Van Cutsem E, Bokemeyer C, Heeger S and Tejpar S: Use of early tumor shrinkage to predict long-term outcome in metastatic colorectal cancer treated with cetuximab. J Clin Oncol 31: 3764-3775, 2013.

3 Rivera F, Karthaus M, Hecht JR and Fasola G: First-line treatment with modified FOLFOX6 + panitumumab or bevacizumab in wild-type RAS metastatic colorectal carcinoma: Tumor response outcomes beyond RECIST. J Clin Oncol 33: abstr 660, 2015.

4 Shindoh J, Loyer EM, Kopetz S, Boonsirikamchai P, Maru DM, Chun YS, Zimmitti G, Curley SA, Charnsangavej C, Aloia TA and Vauthey JN: Optimal morphologic response to preoperative chemotherapy: an alternate outcome end point before resection of hepatic colorectal metastases. J Clin Oncol 30: 4566-4572, 2012.

5 Chun YS, Vauthey JN, Boonsirikamchai P, Maru DM, Kopetz S, Palavecino M, Curley SA, Abdalla EK, Kaur H, Charnsangavej $\mathrm{C}$ and Loyer EM: Association of computed tomography morphologic criteria with pathologic response and survival in patients treated with bevacizumab for colorectal liver metastases. JAMA 302: 2338-2344, 2009.
6 Eisenhauer EA, Therasse P, Bogaerts J, Schwartz LH, Sargent D, Ford R, Dancey J, Arbuck S, Gwyther S, Mooney M, Rubinstein L, Shankar L, Dodd L, Kaplan R, Lacombe D and Verweij J: New response evaluation criteria in solid tumours: revised RECIST guideline (version 1.1). Eur J Cancer 45: 228-247, 2009.

7 Yoshita H, Hosokawa A, Ueda A, Ando T, Kajiura S, Kato H, Kawabe H, Tomizawa G, Horikawa N, Yabuhita K, Note M and Sugiyama T: Predictive value of optimal morphologic response to first-line chemotherapy in patients with colorectal liver metastases. Digestion 89: 43-48, 2014.

8 Miles KA: Tumour angiogenesis and its relation to contrast enhancement on computed tomography: a review. Eur J Radiol 30: 198-205, 1999.

9 Smith AD, Lieber ML and Shah SN: Assessing tumor response and detecting recurrence in metastatic renal cell carcinoma on targeted therapy: importance of size and attenuation on contrastenhanced CT. AJR Am J Roentgenol 194: 157-165, 2010.

10 Smith AD, Shah SN, Rini BI, Lieber ML and Remer EM: Morphology, attenuation, size, and structure (MASS) criteria: assessing response and predicting clinical outcome in metastatic renal cell carcinoma on antiangiogenic targeted therapy. AJR Am J Roentgenol 194: 1470-1478, 2010.

Received September 22, 2018

Revised October 1, 2018

Accepted October 2, 2018 